Fundación

Miguel Lillo

Tucumán

Argentina

\title{
Rescatando la importancia del trabajo botánico de Miguel Lillo (26 de julio de 1862 - 4 de mayo de 1931) en el nonagésimo aniversario de su fallecimiento
}

\begin{abstract}
Highlighting the importance of Miguel Lillo's Botanical Work (July 26, 1862 - May 4, 1931) on the 90th Anniversary of his death
\end{abstract}

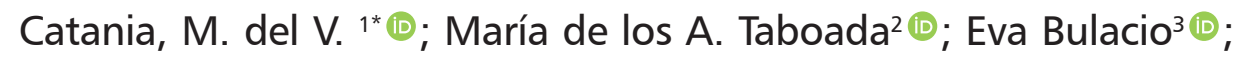

Marcelo D. Arana4 (1)

1 Instituto Criptogámico, Laboratorio de Micología, Fundación Miguel Lillo, Miguel Lillo 251, (T4000JFE) San Miguel de Tucumán, Tucumán, Argentina.

2 Instituto de Ecosistemas de Aguas Continentales, Fundación Miguel Lillo y UEL-CONICET, Miguel Lillo 251, (T4000JFE) San Miguel de Tucumán, Tucumán, Argentina.

3 Instituto de Taxonomía Fanerogámica y Palinología. Fundación Miguel Lillo, Miguel Lillo 251, (T4000JFE) San Miguel de Tucumán, Tucumán, Argentina.

4 Orientación Plantas vasculares, Departamento de Ciencias Naturales, Facultad de Ciencias Exactas, Físico-Químicas y Naturales, Instituto ICBIA (UNRC - CONICET), Universidad Nacional de Río Cuarto, Ruta 36 km 601, (X5804ZAB) Río Cuarto, Córdoba, Argentina.

* Autor corresponsal: mcatania@lillo.org.ar

\section{RESUMEN}

En el nonagésimo aniversario del fallecimiento de Miguel Lillo, el presente trabajo brinda una síntesis de la importancia de las contribuciones botánicas, generadas por una figura emblemática que representa a las Ciencias Naturales en la provincia de Tucumán, Argentina. Se realizó una búsqueda bibliográfica específica sobre Miguel Lillo, biografías y consulta de bases de datos en línea. Se da a conocer una lista de especies descriptas por Lillo validadas actualmente y taxones de plantas y hongos en honor a la memoria del gran sabio.

Palabras clave - Colecciones; Miguel Lillo; Taxonomía; Tucumán.

\footnotetext{
Ref. bibliográfica: Catania, M. del V., Taboada, M. de los A., Bulacio, E., Arana, M. D. (2021). Rescatando la importancia del trabajo botánico de Miguel Lillo (26 de julio de $1862-4$ de mayo de 1931) en el nonagésimo aniversario de su fallecimiento. Lilloa 58 (1): 35-50. doi: https://doi.org/10.30550/j.lil/2021.58 $.1 / 2021.04 .21$

> Recibido: 10 de marzo 2021 - Aceptado: 21 de abril 2021 - Publicado en línea: 27 de abril 2021.

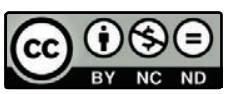

open 2 access > URL de la revista: http://lilloa.lillo.org.ar

- Esta obra está bajo una Licencia Creative Commons Atribución - No Comercial - Sin Obra Derivada 4.0 Internacional.
} 


\begin{abstract}
In the ninetieth anniversary of the death of Miguel Lillo, this paper presents a summary of the important botanical contributions made by such emblematic figure representing the natural sciences in the province of Tucuman, Argentina. A specific bibliographic search of Miguel Lillo's biographies and online database consultations were carried out. A list of currently accepted species described by Lillo along with plant and fungal taxa dedicated in honor of the memory of this great sage is provided.
\end{abstract}

Keywords - Collections; Miguel Lillo; Taxonomy; Tucumán.

\title{
INTRODUCCIÓN
}

En 2021, se conmemora el nonagésimo aniversario del fallecimiento de Miguel Ignacio Lillo (1862-1931), uno de los naturalistas más destacados de Argentina de fines del siglo XIX y comienzos del XX (Fig. 1). Fue, esencialmente, una persona autodidacta, profundamente erudita y dotada de una extraordinaria vocación científica. Especializado en botánica, se dedicó además a otras ramas de la ciencia, en particular la química y la zoología.

Un homenaje es siempre una ocasión de reconocimiento de la labor de quien logró sobresalir por su trabajo, estudio, honorabilidad científica y fundamentalmente, hacia el significado y la trascendencia de su obra que guardan sus memorias y reflejan sus ideales.

Numerosas biografías recorren las diferentes cualidades de la vida del Dr. Miguel Lillo (Castellanos, 1931; Molfino, 1937; Torres, 1958, 2006; Peña de Bascary, 2014).

Miguel Lillo, "el gran naturalista”, fue discípulo del científico Federico Schickendantz (1837, Alemania - 1896, Buenos Aires, Argentina), constituyéndose en su ejemplo y guía. Schickendantz contribuyó en su formación, posibilitando la continuidad de su estudio de las ciencias naturales en Tucumán. Además, Schickendantz fue el nexo de contacto con naturalistas de la Academia Nacional de Ciencias en Córdoba, donde Lillo se relacionó con grandes maestros de la botánica como Federico Kurtz, Jorge Hieronymus, Teodoro Stuckert, Oscar y Adolfo Doering, entre otros. Asimismo, Schickendantz lo incentivó a realizar un viaje a Europa en el año 1897 para completar su formación, en donde conoció a destacados maestros botánicos (Peña de Bascary, 2014). Su estancia en Europa influyó de manera notable en Lillo, a tal punto que llegó a expresar, a su regreso: “...mi resolución está tomada. Mi vocación son las Ciencias Naturales y, entre ellas, la Botánica” (Torres, 1958).

Su pasión por la flora lo llevó a recorrer Tucumán, coleccionando y estudiando las plantas de esta provincia, llegando a formar el herbario más completo del noroeste argentino y publicando luego sus primeros trabajos sobre la flora tucumana (Lillo, $1888,1889)$.

Entre sus principales contribuciones podemos destacar sus aportes al conocimiento de los árboles de la Argentina, que se confeccionó con base en colecciones y 


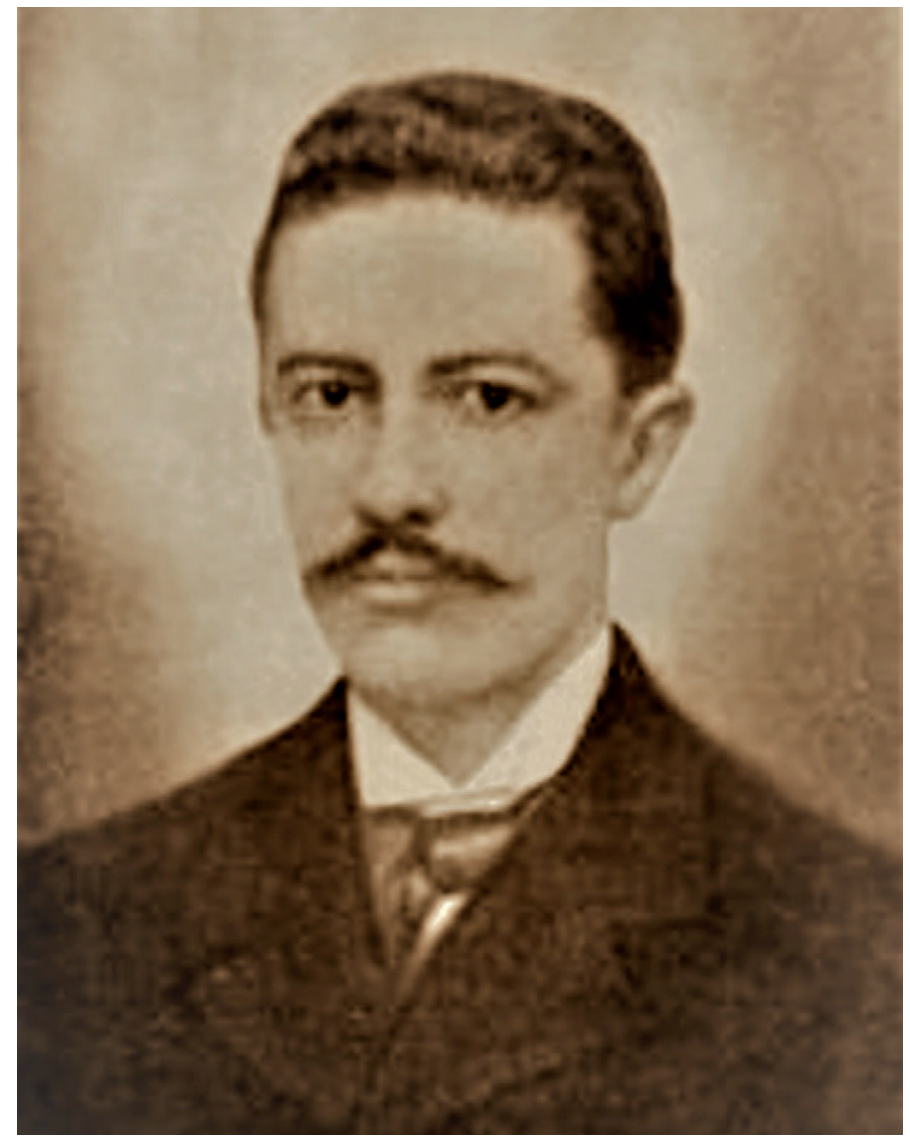

Fig. 1. Retrato de Miguel Lillo. [Disponible en: https://historianatural.wordpress.com/2010/02/14/miguel-lillo-un-naturalistapor-vocacion/]

Fig. 1. Portrait of Miguel Lillo. [Available at: https://historianatural.wordpress.com/2010/02/14/miguel-lillo-un-naturalistapor-vocacion/]

observaciones de Santiago Venturi y determinaciones de Miguel Lillo (Lillo, 1910, 1917) (Fig. 2A). En esta obra constan alrededor de 800 nombres científicos y comunes de árboles del norte y centro de Argentina, además de muestras de madera que recolectó Venturi para la Exposición del Centenario. También aportó al estudio de la familia de las compuestas (Asteraceae), determinando especímenes de varias de sus tribus (Vernonieae, Eupatorieae, Astereae, Inuleae) existentes en los principales herbarios del país y de sus propias colecciones. Asimismo, contribuyó al estudio de las gramíneas (Poaceae) de Tucumán (Lillo, 1916) (Fig. 2B) y de las Asclepiadáceas argentinas (Apocynaceae), enumerando las especies conocidas hasta ese momento, con datos bibliográficos, sinónimos y notas (Lillo, 1919; 1920) (Fig. 3A). Por otro lado, realizó importantes aportes al conocimiento de las Acantáceas (Acanthaceae), resumidos en su manuscrito póstumo publicado en el primer volumen de la revista Lilloa (Lillo, 1937).

Miguel Lillo fue un asiduo viajero y así exploró las distintas regiones de la Argentina, desde Buenos Aires e Isla Martín García, hasta el norte del país, recorriendo la Mesopotamia, Córdoba, Santiago del Estero, Cuyo y Salta. Se destacan 


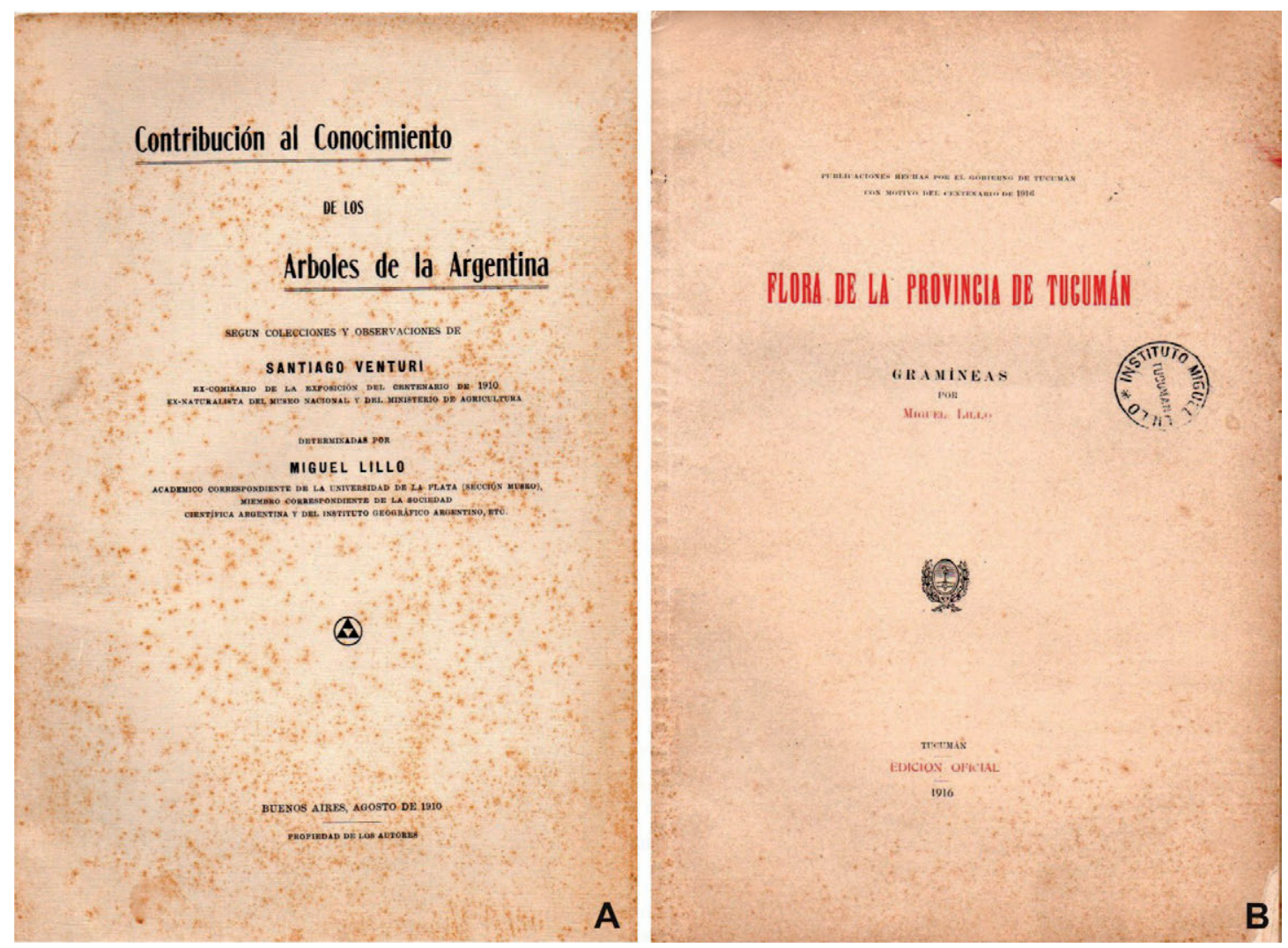

Fig. 2. Publicaciones originales de Miguel Lillo. A) Contribución al conocimiento de los árboles de Argentina. B) Flora de la provincia de Tucumán.

Fig. 2. Original publications of Miguel Lillo. A) Contribution to the knowledge of the trees of Argentina. B) Flora of the province of Tucumán.

principalmente las exploraciones en su provincia natal, con viajes de recolección y reconocimiento de la vegetación (Figs. 4 y 5). Como fruto de los mismos llegó a publicar la distribución de las especies de plantas de Túcumán (Lillo, 1919).

Si bien la botánica era su principal pasión, también ahondó en el estudio de la fauna, colaborando con científicos del país y del extranjero. Como resultado, describió numerosas especies de aves, trabajo que culminó en una publicación sobre la avifauna tucumana (Lillo, 1902) (Fig. 3B), entre otros aportes (Lillo, 1889, 1905, 1909; Dabbene y Lillo, 1913).

Durante muchos años, se entregó con fervor al estudio de la naturaleza, contribuyendo e intercambiando sus conocimientos con los mejores maestros botánicos del país y del extranjero. Fue parte del movimiento botánico argentino en las dos últimas décadas del siglo XIX, momento histórico reconocido como el florecimiento de la ciencia en nuestro país. Durante el transcurso de su carrera recibió numerosos premios y en 1914 la Universidad Nacional de La Plata le otorgó el título de Doctor Honoris Causa.

Noventa años después del paso a la inmortalidad de Miguel Lillo, ejemplo incomparable para los botánicos argentinos, aún se continúa honrando su obra que se encuentra preservada en la biblioteca y Museo de la Fundación Miguel Lillo, y sus invaluables colecciones que se conservan en los herbarios de la Fundación como pa- 


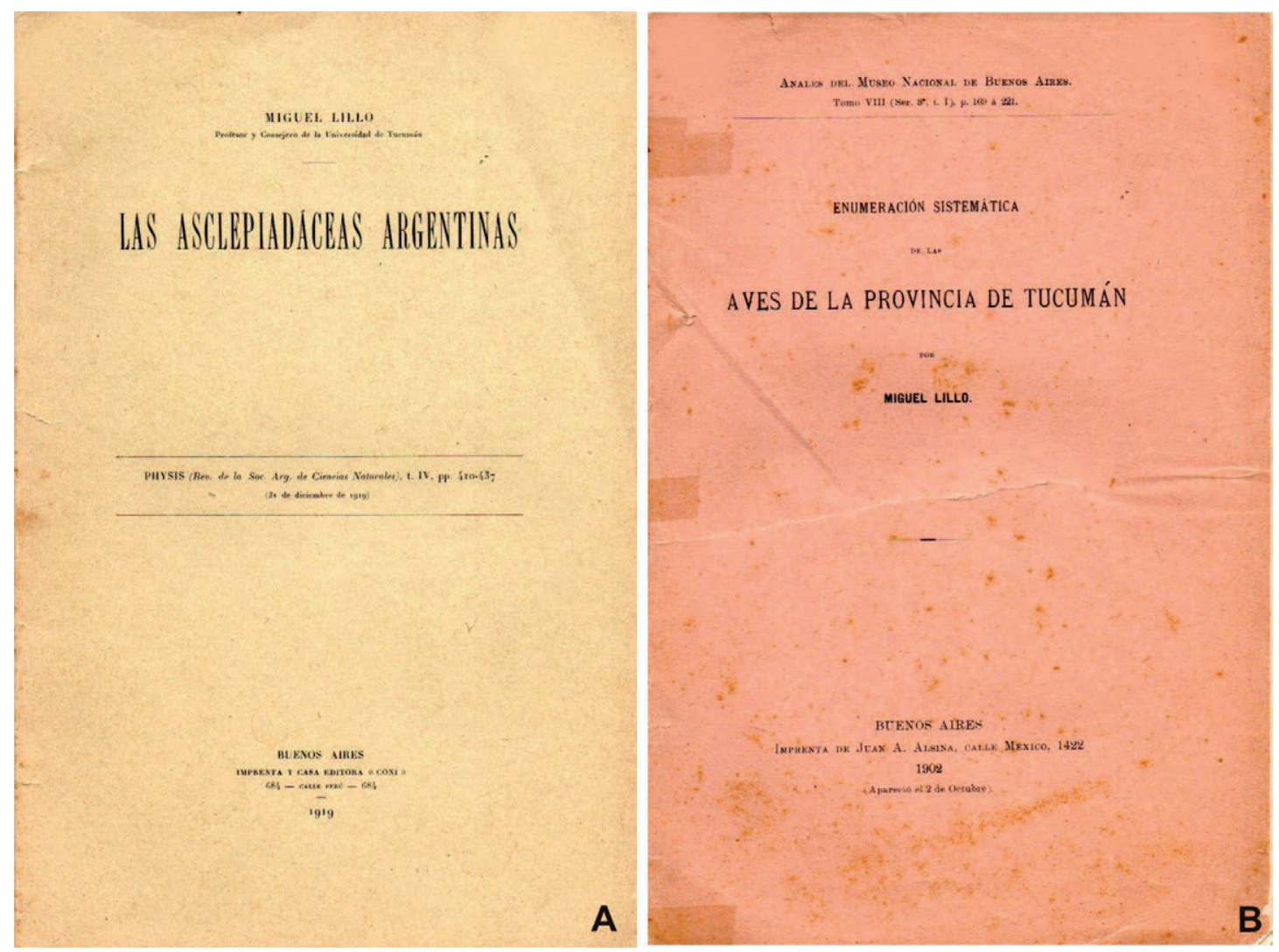

Fig. 3. Publicaciones originales de Miguel Lillo. A) Las Asclepiadáceas Argentinas. B) Aves de la provincia de Tucumán.

Fig. 3. Original publications of Miguel Lillo. A) The Argentine Asclepiads. B) Birds of the Province of Tucumán.

trimonio cultural de primer orden. Uno de los aspectos que destacan la importancia de los aportes de Lillo al conocimiento de la naturaleza argentina, lo constituye la numerosa cantidad de taxones dedicados a él. Debido a esto, en un nuevo aniversario del fallecimiento del gran sabio, el objetivo propuesto en este artículo es realizar una valoración de la importancia de las contribuciones botánicas generadas por el naturalista Miguel Lillo, a través de los taxones de plantas y hongos dedicados en su memoria; así como también brindar un listado de especies descriptas por Lillo, aceptadas actualmente.

\section{MATERIALES Y MÉTODOS}

Para la concreción de este trabajo se llevaron a cabo numerosas búsquedas bibliográficas de diversas fuentes; particularmente se accedió a los archivos de la biblioteca de la Fundación Miguel Lillo, realizando un análisis de los trabajos publicados por él. Asimismo, se consultaron numerosas bases de datos, detalladas a continuación:

https://www.tropicos.org/Name

https://www.ipni.org 

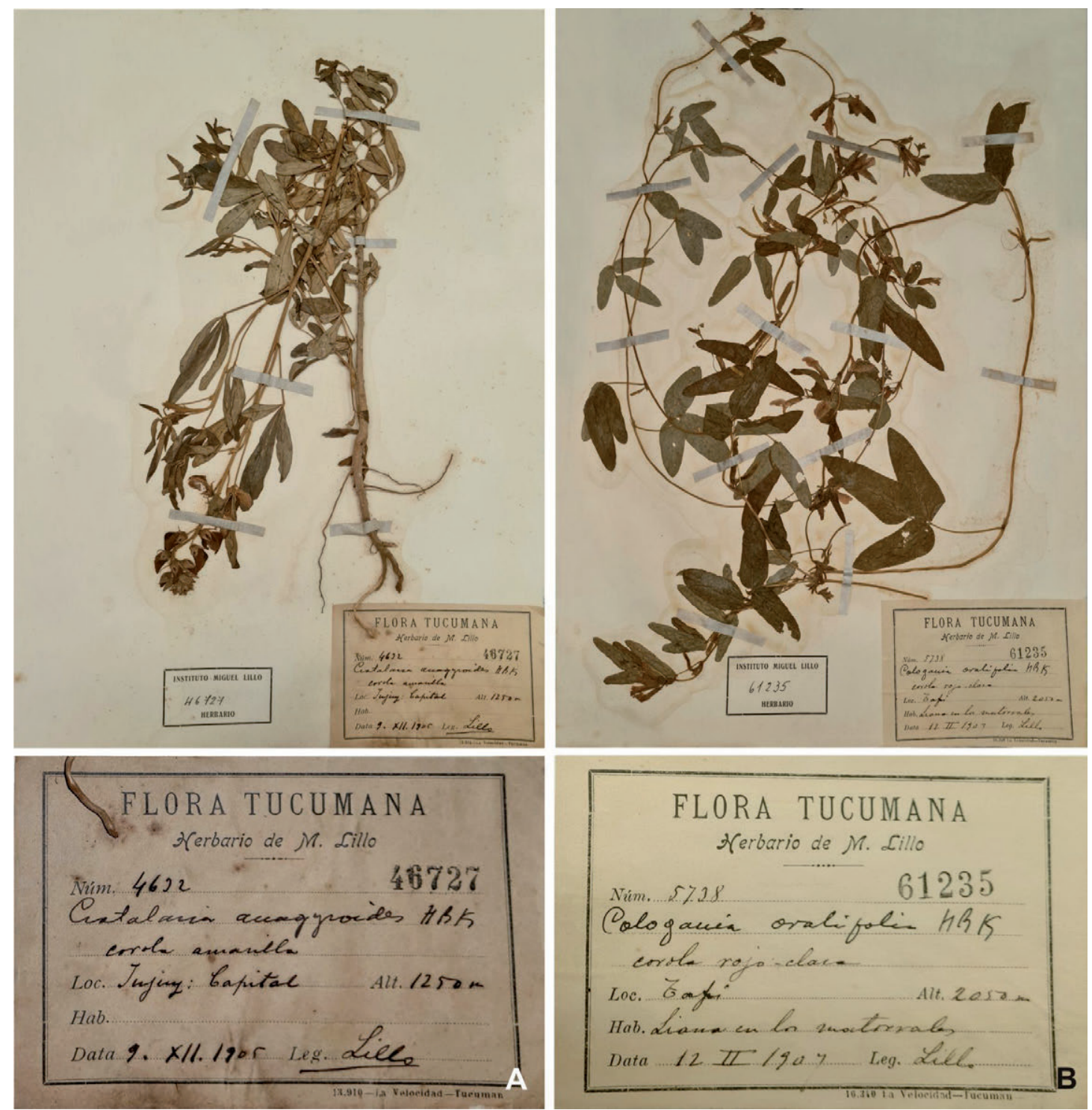

Fig. 4. Ejemplares de herbario recolectados por Miguel Lillo. A) Crotalaria anagyroides Kunth (= Crotalaria micans Link, Fabaceae). B) Cologania ovalifolia Kunth (= Cologania broussonetii (Balb.) DC., Fabaceae). [Herbario LIL].

Fig. 4. Miguel Lillo's Herbarium specimens. A) Crotalaria anagyroides Kunth (= Crotalaria micans Link, Fabaceae). B) Cologania ovalifolia Kunth (= Cologania broussonetii (Balb.) DC., Fabaceae). [LIL Herbarium].

http://www.darwin.edu.ar/Proyectos/FloraArgentina/DetalleEspecie http://www.indexfungorum.org/names/names.asp

https://www.mycobank.org/account/login

\section{RESULTADOS}

A manera de homenaje, numerosos investigadores le dedicaron y dedican al Dr. Miguel Lillo, taxones nuevos (géneros, especies, subespecies y variedades), llegando a enumerarse, hasta el momento (considerando plantas y hongos), un género y 


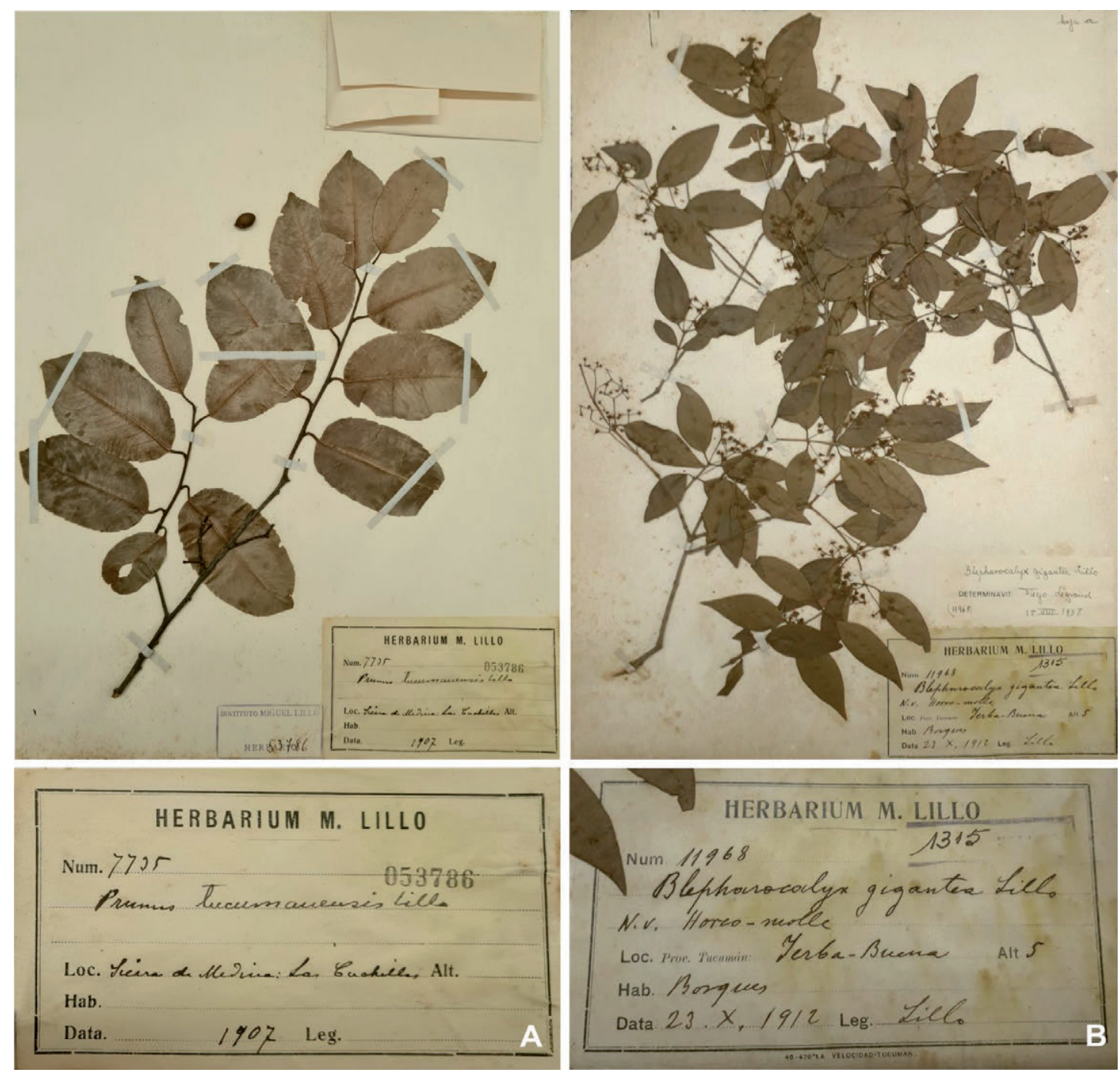

Fig. 5. Especies colectadas y descriptas por Miguel Lillo. A) Prunus tucumanensis Lillo (Rosaceae). B) Blepharocalyx gigantea Lillo (Myrtaceae). [Herbario LIL].

Fig. 5. Collected and described species by Miguel Lillo. A) Prunus tucumanensis Lillo (Rosaceae). B) Blepharocalyx gigantea Lillo (Myrtaceae). [LIL Herbarium].

otros 55 taxones propuestos por diferentes autores, de los cuales 30 están aceptados actualmente (Tabla 1, Figs. 6 y 7).

Por otro lado, se presenta un listado de algunas de las numerosas especies que el Dr. Lillo describió a lo largo de su extensa trayectoria profesional (Tabla 2, Fig. 8).

\section{Género}

Lilloa Speg., Pl. Nov. Argent. 3.: 10. 1896.

Este género, propuesto por el botánico italiano Carlos Spegazzini y perteneciente a la familia de las Araceae, fue considerado poco después como sinónimo de Synandrospadix Engl. (Hauman y Vandenverken, 1917; Hicken, 1922).

\section{Especies y taxones infraespecíficos}


Tabla 1. Taxones específicos e infraespecíficos dedicados a Miguel Lillo, ordenados alfabéticamente, con la publicación original, la familia y su estatus taxonómico actual. Los nombres aceptados actualmente se señalan en negrita.

Table 1. Specific and infraspecific taxa dedicated to Miguel Lillo, arranged alphabetically, with original publication, family and current status. Currently accepted names are in bold.

\begin{tabular}{|c|c|c|c|c|}
\hline & Especie & Publicación & Familia & Estatus \\
\hline \multirow{13}{*}{$\frac{\amalg}{\longleftarrow}$} & Abutilon lilloi Hassl. & $\begin{array}{l}\text { Repert. Spec. Nov. } \\
\text { Regni Veg. 12: } 497 . \\
1913 .\end{array}$ & Malvaceae & $\begin{array}{l}\text { Sinónimo de Abutilon } \\
\text { thyrsodendron Griseb., } \\
\text { Abh. Königl. Ges. Wiss. } \\
\text { Göttingen 24: 48. } 1879 .\end{array}$ \\
\hline & $\begin{array}{l}\text { Acaena polycarpa subsp. lilloi } \\
\text { Bitter }\end{array}$ & $\begin{array}{l}\text { Repert. Spec. Nov. } \\
\text { Regni Veg. 12: } 480 . \\
1913 .\end{array}$ & Rosaceae & $\begin{array}{l}\text { Sinónimo de Acaena } \\
\text { stricta Griseb., Abh. } \\
\text { Königl. Ges. Wiss. } \\
\text { Göttingen 19: } 138 . \\
1874 .\end{array}$ \\
\hline & $\begin{array}{l}\text { Allophylus pauciflorus Radlk.var. } \\
\text { lilloi F.A. Barkley }\end{array}$ & $\begin{array}{l}\text { Lilloa 28: } 166 . \\
1957 .\end{array}$ & Sapindaceae & $\begin{array}{l}\text { Sinónimo de Allophylus } \\
\text { pauciflorus Radlk., } \\
\text { Meded. Rijks-Herb. 19: } \\
61.1913 .\end{array}$ \\
\hline & Asplenium lilloanum de la Sota & $\begin{array}{l}\text { Darwiniana 18: } 198, \\
\text { f.1,7b. } 1973 \text {. }\end{array}$ & Aspleniaceae & Aceptado \\
\hline & Atriplex lilloi Hauman & $\begin{array}{l}\text { Anales Mus. Nac. } \\
\text { Hist. Nat. Buenos } \\
\text { Aires 32: 84. } 1923 . \\
1925 .\end{array}$ & Amaranthaceae & $\begin{array}{l}\text { Sinónimo de Atriplex } \\
\text { myriophylla Phil., Anales } \\
\text { Mus. Nac. Chile, Secc. 2, } \\
\text { Bot. 8: } 74.1891 .\end{array}$ \\
\hline & Baccharis lilloi Heering & $\begin{array}{l}\text { Jahrb. Hamburg. } \\
\text { Wiss. Anst. 31, } \\
\text { Beiheft 3: } 78.1915 .\end{array}$ & Asteraceae & Aceptado \\
\hline & Berberis lilloana Job & Lilloa 17: 82. 1949. & Berberidaceae & Aceptado \\
\hline & Briza lilloi Parodi & $\begin{array}{l}\text { Revista Fac. Agron. } \\
\text { Veterin. 3: 133, figs. } \\
6,7.1920 .\end{array}$ & Poaceae & $\begin{array}{l}\text { Sinónimo de } \\
\text { Chascolytrum } \\
\text { monandrum (Hack.) } \\
\text { Essi, Longhi-Wagner \& } \\
\text { Souza-Chies, Novon 21: } \\
\text { 329. } 2011 .\end{array}$ \\
\hline & Calamagrostis lilloi Hack. & $\begin{array}{l}\text { Anales Mus. Nac. } \\
\text { Buenos Aires ser. 3, } \\
6: 477.1906 .\end{array}$ & Poaceae & $\begin{array}{l}\text { Sinónimo de } \\
\text { Cinnagrostis polygama } \\
\text { Griseb. var. polygama, } \\
\text { Abh. Königl. Ges. Wiss. } \\
\text { Göttingen 19: } 257, \text { t. } 2 \text {, } \\
\text { f. } 7.1874 \text {. }\end{array}$ \\
\hline & Calceolaria lilloi Descole \& Borsini & Lilloa 27: 75. 1955. & Calceolariaceae & $\begin{array}{l}\text { Sinónimo de Calceolaria } \\
\text { chelidonioides Kunth, } \\
\text { Nov. Gen. Sp. (quarto } \\
\text { ed.) 2: } 378.1817 \\
\text { [1818]. }\end{array}$ \\
\hline & Calyptranthes lilloi Speg. & $\begin{array}{l}\text { Cat. Descr. Maderas } \\
\text { 386. } 1910 .\end{array}$ & Myrtaceae & $\begin{array}{l}\text { Basónimo de Myrcia } \\
\text { lilloi (Speg.) E.Lucas } \\
\text { \& K.Samra, Phytotaxa } \\
\text { 406: 149. } 2019 .\end{array}$ \\
\hline & Cedrela lilloi C.DC. & $\begin{array}{l}\text { Bull. Soc. Bot. } \\
\text { Genève ser. } 2 \text {, } 6 \text { : } \\
\text { 118, fig. 2. } 1914 .\end{array}$ & Meliaceae & $\begin{array}{l}\text { Sinónimo de Cedrela } \\
\text { angustifolia DC., Prodr. } \\
\text { 1: } 624.1824 .\end{array}$ \\
\hline & Chaetochlamys lilloi J. L. Lotti & $\begin{array}{l}\text { Publ. Espec. Inst. } \\
\text { Lillo 1187: } 63 . \\
1976 .\end{array}$ & Acanthaceae & $\begin{array}{l}\text { Basónimo de Justicia } \\
\text { Iilloi (Lotti) C. Ezcurra, } \\
\text { FI. Prov. Jujuy, Colecc. } \\
\text { Ci. Inst. Nac. Tecnol. } \\
\text { Agropecu. 13(9): } 349 . \\
1993 .\end{array}$ \\
\hline
\end{tabular}


Tabla 1 (continuación).

Table 1 (continued).

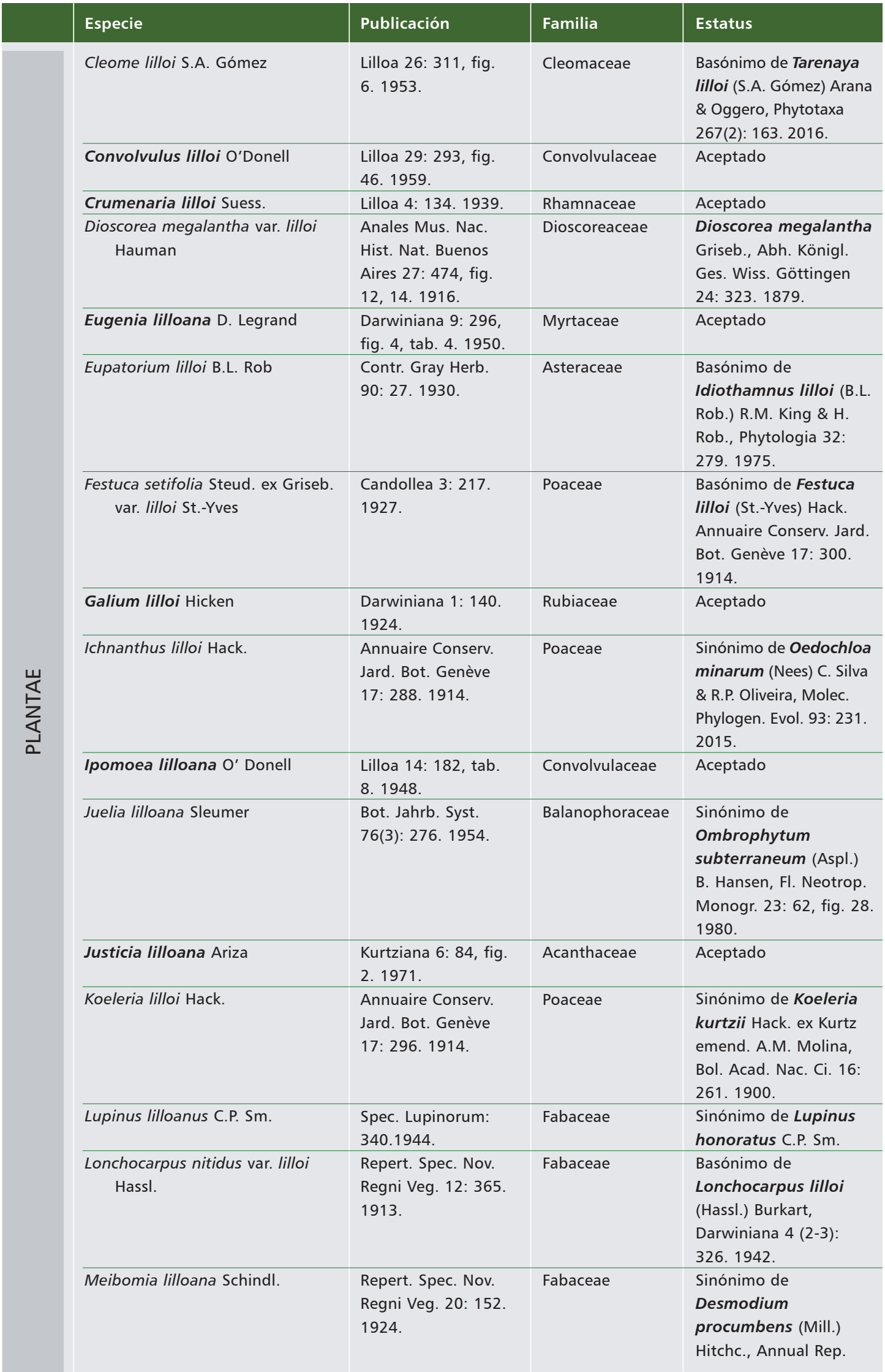


Tabla 1 (continuación).

Table 1 (continued).

\begin{tabular}{|c|c|c|c|c|}
\hline & Especie & Publicación & Familia & Estatus \\
\hline \multirow{15}{*}{$\frac{m}{\frac{\pi}{5}}$} & & & & $\begin{array}{l}\text { Missouri Bot. Gard. 4: } \\
\text { 76. } 1893 .\end{array}$ \\
\hline & Melica Iilloi Bech. & $\begin{array}{l}\text { Candollea 7: } 520 . \\
1938 .\end{array}$ & Poaceae & Aceptado \\
\hline & Mitostigma lilloi T. Mey. & $\begin{array}{l}\text { Lilloa } 6: 340, \text { tab. } 1 . \\
1941 .\end{array}$ & Apocynaceae & $\begin{array}{l}\text { Sinónimo de Philibertia } \\
\text { coalita (Lillo) Goyder, } \\
\text { Kew Bull. 59(3): } 445 . \\
2004 \text {. }\end{array}$ \\
\hline & Nephrodium lilloi Hicken & $\begin{array}{l}\text { Anales Soc. Ci. } \\
\text { Argent. 62: } 166 . \\
1966 .\end{array}$ & Athyriaceae & $\begin{array}{l}\text { Basónimo de Diplazium } \\
\text { lilloi (Hicken) R.M. } \\
\text { Tryon \& A. F. Tryon, } \\
\text { Rhodora 84: 127. } 1982 .\end{array}$ \\
\hline & Oxalis lilloana R. Knuth & $\begin{array}{l}\text { Repert. Spec. Nov. } \\
\text { Regni Veg. 23: } 140 . \\
1926 .\end{array}$ & Oxalidaceae & $\begin{array}{l}\text { Sinónimo de Oxalis } \\
\text { barrelieri L., Sp. PI. ed. } \\
\text { 2: } 624.1762 .\end{array}$ \\
\hline & Oxalis lilloi Hicken & $\begin{array}{l}\text { Darwiniana 1: } 125 . \\
1924 .\end{array}$ & Oxalidaceae & $\begin{array}{l}\text { Sinónimo de Oxalis } \\
\text { argentina R. Knuth, } \\
\text { Notizbl. Bot. Gart. } \\
\text { Berlin-Dahlem } 7 \text { (67): } \\
\text { 313. } 1919 .\end{array}$ \\
\hline & Panicum lilloi Stuck. & $\begin{array}{l}\text { Anales Mus. Nac. } \\
\text { Buenos Aires 13: } \\
\text { 432. } 1906 .\end{array}$ & Poaceae & $\begin{array}{l}\text { Setaria oblongata } \\
\text { (Griseb.) Parodi, Physis } \\
\text { (Buenos Aires) 9: 13, } \\
\text { 38. } 1928 .\end{array}$ \\
\hline & Paspalum lilloi Hack. & $\begin{array}{l}\text { Annuaire Conserv. } \\
\text { Jard. Bot. Genève } \\
\text { 17: } 282.1914 .\end{array}$ & Poaceae & Aceptado \\
\hline & Pellaea lilloi Hicken & $\begin{array}{l}\text { Anales Soc. Ci. } \\
\text { Argent. 62: } 210 . \\
1906 .\end{array}$ & Pteridaceae & $\begin{array}{l}\text { Sinónimo de } \\
\text { Cheilanthes squamosa } \\
\text { Gillies ex Hook. \& Grev., } \\
\text { Icon. Filic. tab. } 151 . \\
1831 .\end{array}$ \\
\hline & Peperomia lilloi C.DC. & $\begin{array}{l}\text { Repert. Spec. Nov. } \\
\text { Regni Veg. 14: } 397 . \\
1916 .\end{array}$ & Piperaceae & $\begin{array}{l}\text { Sinónimo de Peperomia } \\
\text { santaelisae C. DC., Bull. } \\
\text { Herb. Boissier (sér. 2) 7: } \\
\text { 917. } 1907 .\end{array}$ \\
\hline & $\begin{array}{l}\text { Pisonia ambigua Heimerl var. } \\
\text { lilloana Heimerl }\end{array}$ & $\begin{array}{l}\text { Annuaire Conserv. } \\
\text { Jard. Bot. Genève } \\
\text { 17: } 232.1913 .\end{array}$ & Nyctaginaceae & $\begin{array}{l}\text { Sinónimo de Pisonia } \\
\text { ambigua Heimerl, } \\
\text { Denkschr. Kaiserl. Akad. } \\
\text { Wiss., Math.-Naturwiss. } \\
\text { KI. 79: 236. 1988. }\end{array}$ \\
\hline & Poa lilloi Hack. & $\begin{array}{l}\text { Anales Mus. Nac. } \\
\text { Hist. Nat. Buenos } \\
\text { Aires ser. 3, 14: } \\
\text { 153.1911. }\end{array}$ & Poaceae & Aceptado \\
\hline & Puya Iilloi A. Cast. & $\begin{array}{l}\text { Anales Mus. Nac. } \\
\text { Hist. Nat. Buenos } \\
\text { Aires 36: 54, fig. } 7 . \\
1929 .\end{array}$ & Bromeliaceae & Aceptado \\
\hline & Senecio lilloi Cabrera & $\begin{array}{l}\text { Notas Mus. La Plata, } \\
\text { Bot. 1: 399, fig. } 3 . \\
1936 .\end{array}$ & Asteraceae & Aceptado \\
\hline & Sida lilloana Rodrigo & Lilloa 8: 437. 1942. & Malvaceae & $\begin{array}{l}\text { Sinónimo de Sida } \\
\text { argentina K. Schum. } \\
\text { var. argentina, Fl. Bras. } \\
12 \text { (3): } 315.1891 .\end{array}$ \\
\hline
\end{tabular}


Tabla 1 (continuación).

Table 1 (continued).

\begin{tabular}{|c|c|c|c|c|}
\hline & Especie & Publicación & Familia & Estatus \\
\hline \multirow{7}{*}{ 崖 } & Stevia lilloi B.L. Rob & $\begin{array}{l}\text { Contr. Gray Herb. } \\
90: 14,64.1930 .\end{array}$ & Asteraceae & Aceptado \\
\hline & Stipa caespitosa var. lilloi Hack. & $\begin{array}{l}\text { Anales Mus. Nac. } \\
\text { Hist. Nat. Buenos } \\
\text { Aires ser. 3, 14: } 72 . \\
1911 .\end{array}$ & Poaceae & $\begin{array}{l}\text { Sinónimo de Nassella } \\
\text { inconspicua (J. Presl) } \\
\text { Barkworth, Taxon } 39 \\
\text { (4): } 610.1990 .\end{array}$ \\
\hline & Tournefortia lilloi I.M. Johnst. & $\begin{array}{l}\text { Contr. Gray Herb. } \\
\text { 92: } 71.1930 .\end{array}$ & Boraginaceae & $\begin{array}{l}\text { Basónimo de } \\
\text { Heliotropium Iilloi } \\
\text { (I.M. Johnst.) J.I. M. } \\
\text { Melo, Harvard Pap. Bot. } \\
\text { 23: 15. } 1018 .\end{array}$ \\
\hline & Trichloris lilloi Parodi & $\begin{array}{l}\text { Physis (Buenos } \\
\text { Aires) 9: 256. } 1928 .\end{array}$ & Poaceae & $\begin{array}{l}\text { Sinonimo de } \\
\text { Leptochloa pluriflora } \\
\text { (E. Fourn.) P.M. Peterson } \\
\text { \& N.W. Snow, Ann. Bot. } \\
\text { (Oxford) n.s. 109: } 1327 . \\
2012 .\end{array}$ \\
\hline & $\begin{array}{l}\text { Urtica magellanica var. lilloi } \\
\text { Hauman }\end{array}$ & $\begin{array}{l}\text { Anales Mus. Nac. } \\
\text { Hist. Nat. Buenos } \\
\text { Aires 32: 415. } 1925 .\end{array}$ & Urticaceae & $\begin{array}{l}\text { Basónimo de Urtica } \\
\text { lilloi (Hauman) Geltman, } \\
\text { Novon 8: 17. } 1998 .\end{array}$ \\
\hline & Verbena lilloana Moldenke & $\begin{array}{l}\text { Phytologia 3: } 176 . \\
1949 .\end{array}$ & Verbenaceae & $\begin{array}{l}\text { Basónimo de } \\
\text { Glandularia lilloana } \\
\text { (Moldenke) Botta, Bol. } \\
\text { Soc. Argent. Bot. 26: } \\
\text { 244. 1990. }\end{array}$ \\
\hline & Verbesina lilloi S.F. Blake & $\begin{array}{l}\text { Amer. J. Bot. 12: } \\
\text { 633. } 1925 .\end{array}$ & Asteraceae & Aceptado \\
\hline \multirow{5}{*}{$\stackrel{\bar{\Xi}}{Z}$} & Amanita lilloi Singer & $\begin{array}{l}\text { Lilloa 25: } 245 \\
\text { (1952) [1951]. }\end{array}$ & Amanitaceae & $\begin{array}{l}\text { Basónimo de } \\
\text { Saproamanita lilloi } \\
\text { (Singer) Redhead, } \\
\text { Vizzini, Drehmel \& } \\
\text { Contu, IMA Fungus } 7 \\
\text { (1): } 123 \text { (2016). }\end{array}$ \\
\hline & Geastrum lilloi L.S. Domínguez & $\begin{array}{l}\text { Mycología } 88 \text { (5): } \\
858 \text { (1996). }\end{array}$ & Geastraceae & Aceptado \\
\hline & $\begin{array}{l}\text { Hypoxylon lilloi Sir, C. Lamb. \& } \\
\text { Kuhnert }\end{array}$ & $\begin{array}{l}\text { Fungal Diversity 78: } \\
118 \text { (2016). }\end{array}$ & Hypoxylaceae & Aceptado \\
\hline & Dictyophora lilloi Speg. & $\begin{array}{l}\text { Anal. Mus. nac. B. } \\
\text { Aires Ser. 3, } 16 \text { (9): } \\
30 \text { (1906). }\end{array}$ & Phallaceae & Aceptado \\
\hline & Uredo lilloi Speg. & $\begin{array}{l}\text { Anal. Mus. nac. } \\
\text { Hist. nat. B. Aires 6: } \\
234 \text { (1898) [1899]. }\end{array}$ & Uropyxidaceae & $\begin{array}{l}\text { Sinónimo de Uredo } \\
\text { cuticulosa Ellis \& } \\
\text { Everh., Bull. Lab. Nat. } \\
\text { Hist. Iowa 4: 67. } 1896 .\end{array}$ \\
\hline
\end{tabular}



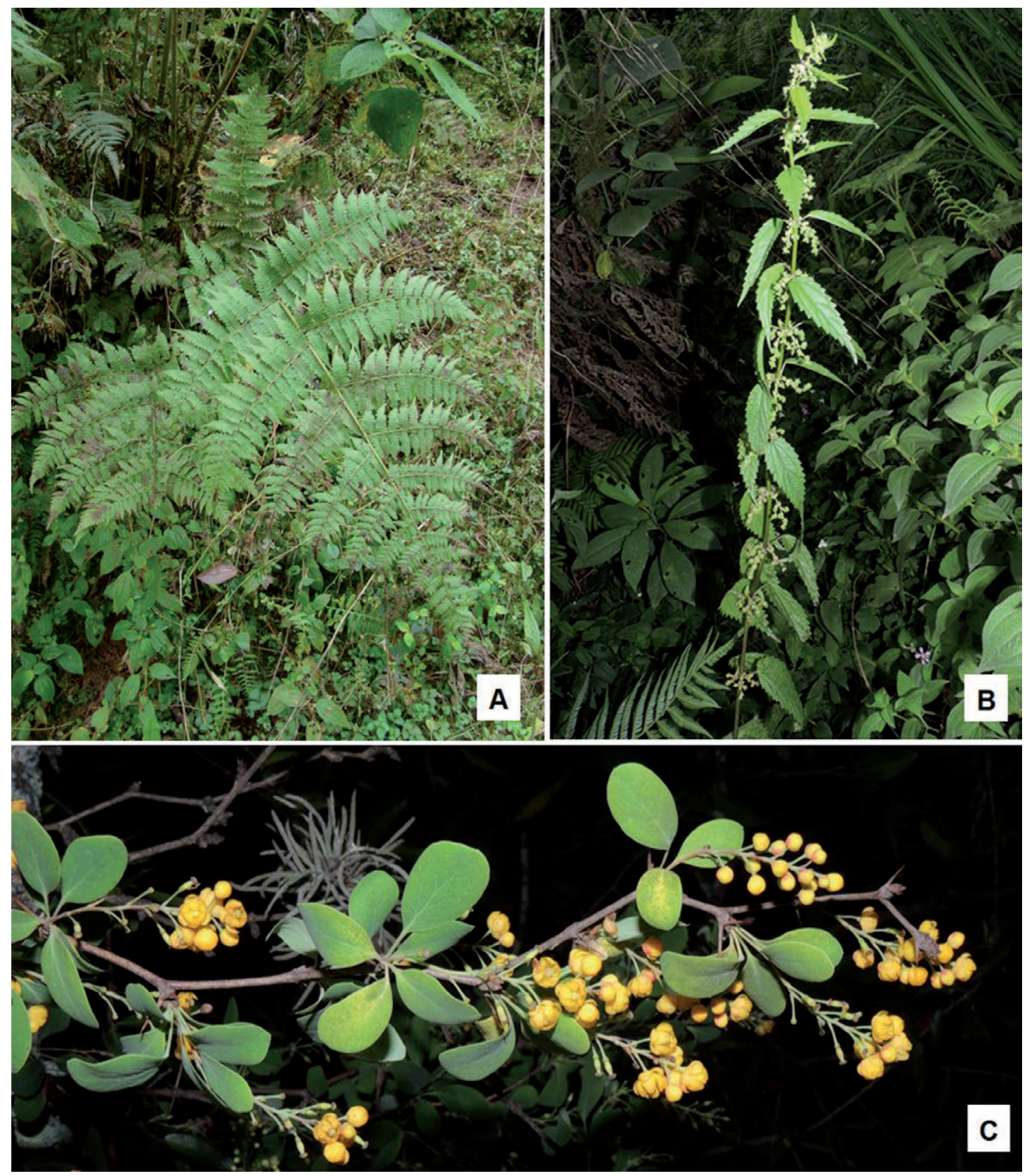

Fig. 6. Especies dedicadas a Miguel Lillo. A) Diplazium lilloi (Hicken) R. M. Tryon \& A. F. Tryon. B) Urtica lilloi (Hauman) Geltman. C) Berberis lilloana Job. Fotografías: A) M. Arana. B y C) E. Bulacio.

Fig. 6. Species dedicated to Miguel Lillo. A) Diplazium lilloi (Hicken) R. M. Tryon \& A. F. Tryon. B) Urtica lilloi (Hauman) Geltman. C) Berberis lilloana Job. Photos: A) M. Arana. B y C) E. Bulacio. 
Tabla 2. Listado de especies seleccionadas descriptas por Lillo, con su correspondiente familia y estatus taxonómico actual. Los nombres aceptados actualmente se señalan en negrita.

Table 2. List of selected species described by Lillo, with their corresponding family and current taxonomic status. Currently accepted names are in bold.

\begin{tabular}{l|l|l} 
Especie & Familia & Estatus \\
\hline Abatia stellata & Salicaceae & Aceptado \\
\hline Basanacantha micrantha & Rubiaceae & $\begin{array}{l}\text { (= Randia micrantha (Lillo) } \\
\text { Bacigalupo var. micrantha) }\end{array}$ \\
\hline Blepharocalyx gigantea & Myrtaceae & $\begin{array}{l}\text { (= Blepharocalyx salicifolius } \\
\text { (Kunth) O. Berg. }\end{array}$ \\
\hline Blepharocalyx montanus & Myrtaceae & $\begin{array}{l}\text { (= Blepharocalyx salicifolius } \\
\text { (Kunth) O. Berg. }\end{array}$ \\
\hline Ilex argentina & Aquifoliaceae & Aceptado \\
\hline Prunus tucumanensis & Rosaceae & Aceptado \\
\hline Crinodendron tucumanun & Elaeocarpaceae & Aceptado \\
\hline Mitostigma castillonii & Apocynaceae & $\begin{array}{l}\text { (= Philibertia castillonii (Lillo) } \\
\text { Goyder. }\end{array}$ \\
\hline Solanum abutiloides (Griseb.) Bitter \& Lillo & Solanaceae & Aceptado \\
\hline Solanum argentinum Bitter \& Lillo & Solanaceae & Aceptado \\
\hline Fagara coco (Gillies) Engl. var. formosana & Rutaceae & $\begin{array}{l}\text { (=Zantoxylum petiolare A. St. } \\
\text { Hill. \& Tul. }\end{array}$ \\
\hline Trechonaetes rotacea & & $\begin{array}{l}\text { (= Jaborosa rotacea (Lillo) Hunz. } \\
\text { \& Barboza. }\end{array}$ \\
\hline Tagetes anisata & Solanaceae & (= Tagetes filifolia Lag.). \\
\hline
\end{tabular}
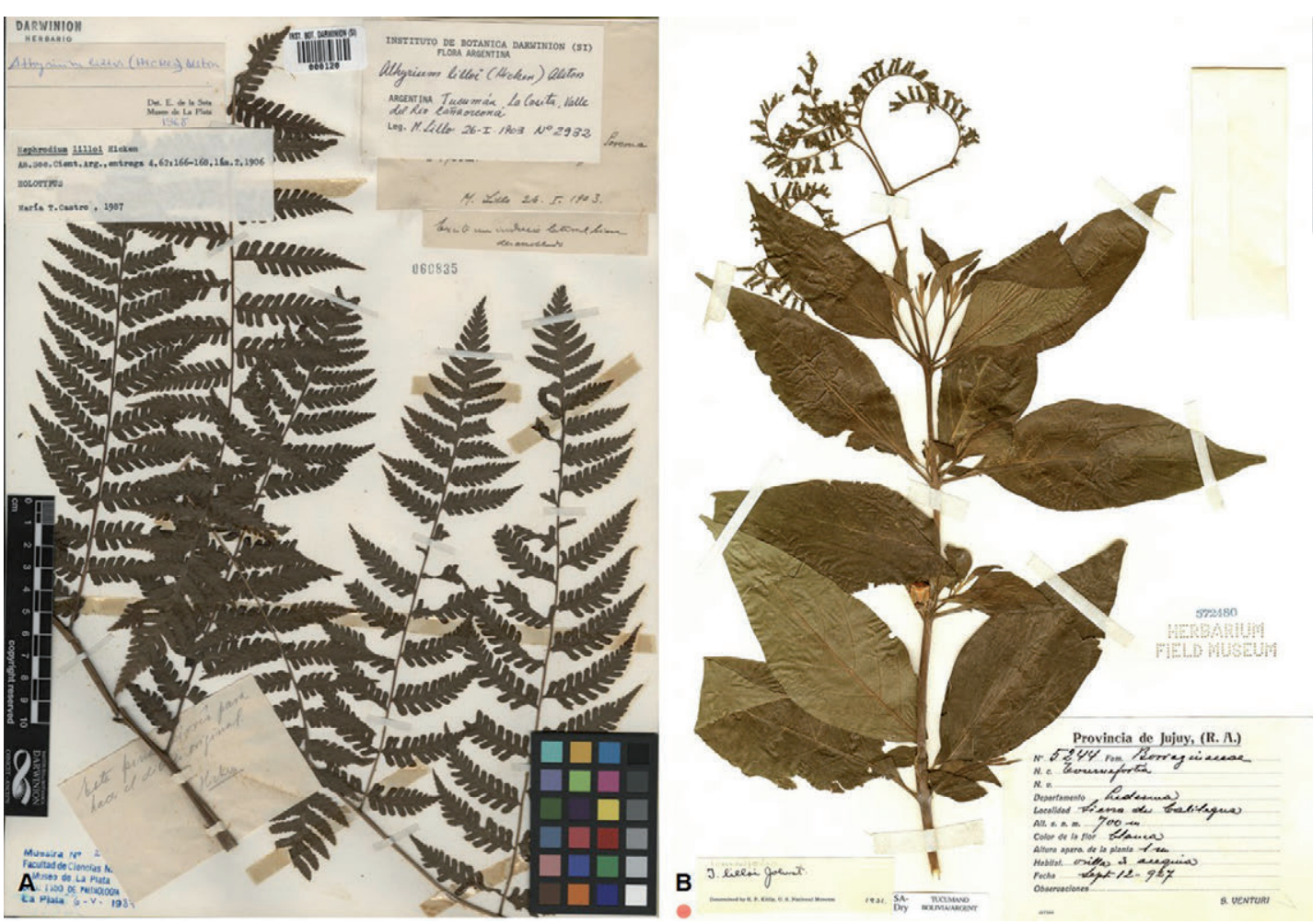

Fig. 7. Ejemplar de herbario nominado en honor a Miguel Lillo. A) Nephrodium lilloi Hicken (Athyriaceae), [http://www2.darwin.edu.ar/ImagenesIris/SI000120.jpg]. B) Tournefortia lilloi I.M. Johnst. (Boraginaceae), [https://collections-botany.fieldmuseum.org/]

Fig. 7. Herbarium specimen named in Miguel Lillo's honor. A) Nephrodium lilloi Hicken (Athyriaceae), [http://www2.darwin.edu.ar/ImagenesIris/SI000120.jpg] B) Tournefortia lilloi I.M. Johnst. (Boraginaceae), [https://collections-botany.fieldmuseum.org/] 


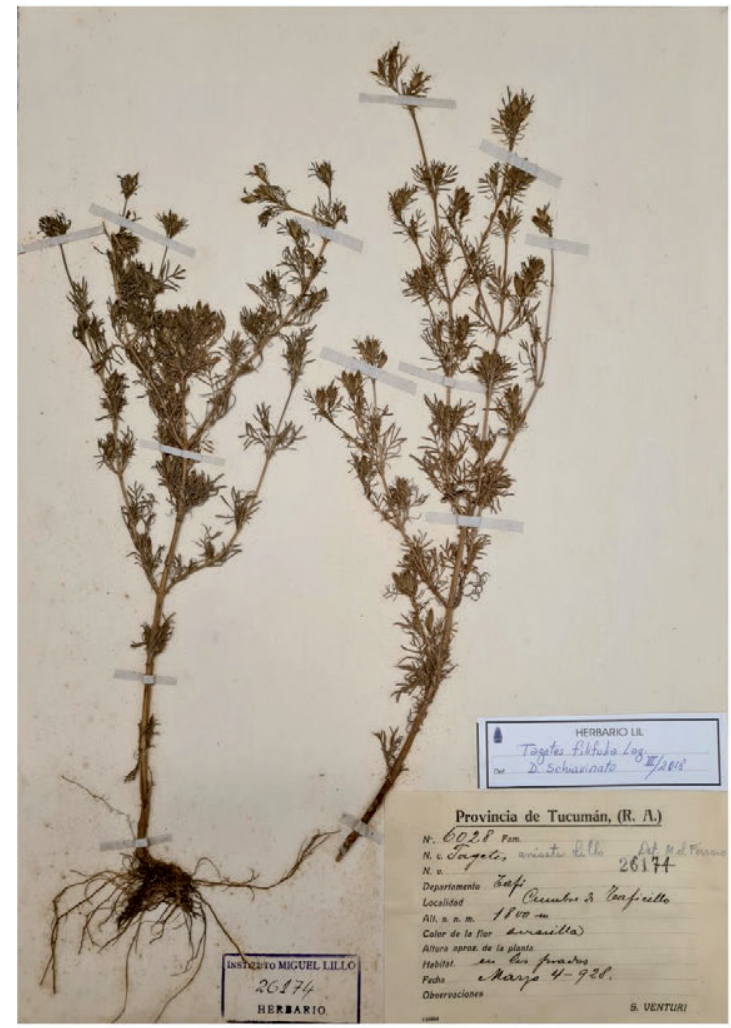

Provincia de Tucumán, (R. A.)

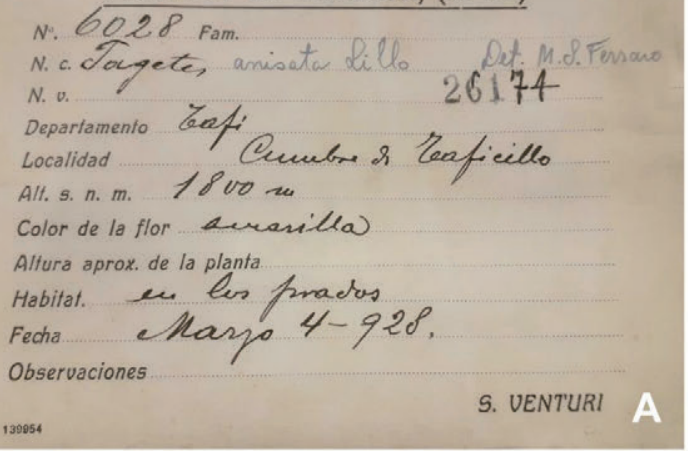

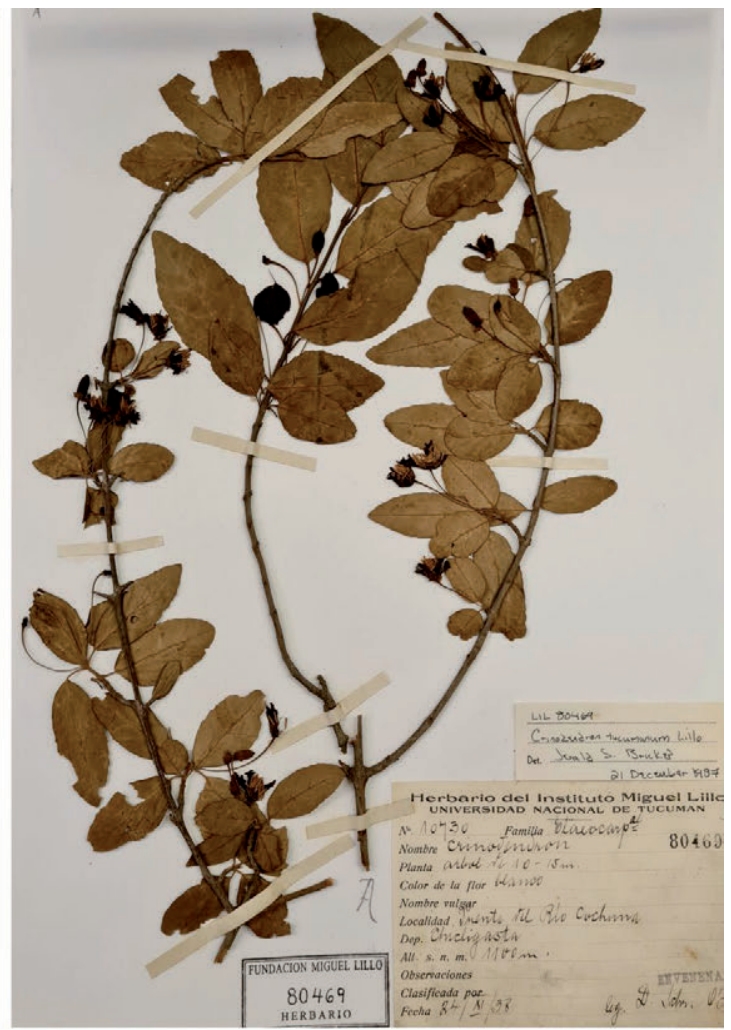

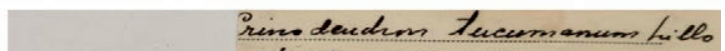
Lieles $\pi(1538) 351$ Det. Denele, o-Donee

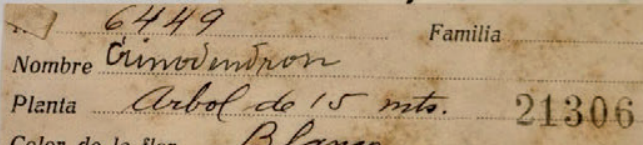

Planta Cubof de

Color de la flor Blaneo

Nombre vulgar

Localidad Las Lenquas Pio Cortuma a Dep - Esquina raude- Isp. Cidilijasta Alt.s.n.m. 1 soo sut.

Observaciones

Clasificada por

Fecha $/ / x / 1930$

Fig. 8. Especies descriptas por M. Lillo, colectadas por Venturi y Schreiter. A) Tagetes anisata Lillo (Asteraceae). B) Crinodendron tucumanum Lillo (Elaeocarpaceae). [Herbario LIL].

Fig. 8. Species described by M. Lillo, collected by Venturi and Schreiter. A) Tagetes anisata Lillo (Asteraceae). B) Crinodendron tucumanum Lillo (Elaeocarpaceae). [LIL Herbarium].

\section{CONSIDERACIONES FINALES}

Este trabajo pretende aportar un reconocimiento más a la trayectoria y memoria del sabio y naturalista Miguel Lillo, desde una perspectiva botánica, teniendo en cuenta los taxones dedicados a su memoria y algunos de su propia autoría. Sus aportes en el grupo de las compuestas (Asteraceae), las asclepiadáceas (Apocynaceae) y las gramíneas (Poaceae) fueron pioneros en su época, y se constituyeron en el inicio de líneas de investigación que continúan en la actualidad. Su trabajo acerca de los árboles de Tucumán fue una contribución destacable, y que se sigue actualizando año a año. 
Su extenso intercambio de cartas y de material colectado con botánicos argentinos y extranjeros fue de suma importancia para enriquecer las colecciones y potenciar el trabajo taxonómico entre él y sus colegas; como corolario, el herbario LIL es uno de los más completos de Argentina.

Como se puede apreciar por el gran número de taxones que inmortalizan su memoria (30 en total, entre plantas vasculares y hongos) su relevancia y su impronta es mundialmente reconocida por múltiples investigadores. En la búsqueda de información se pudo apreciar que las especies descriptas por el Dr. Miguel Lillo en sus publicaciones son más de cien, de manera que su trabajo fue detallado y preciso, lo que acrecentó considerablemente el conocimiento de la biodiversidad vegetal en el país.

La Fundación Miguel Lillo, creada luego del fallecimiento del naturalista, custodia su legado y alberga sus colecciones, en las cuales se encuentra el testimonio de una gran parte de la historia de la tierra, como así también el trabajo y dedicación de muchas generaciones de científicos, técnicos y funcionarios que se esforzaron en la conservación del patrimonio lilloano y que representan un orgullo para la provincia y el país.

En este 90 aniversario de su partida solo nos queda agradecer y valorar su legado y continuar acrecentándolo, formando botánicos/as que continúen en el futuro con la ardua tarea de clasificar, estudiar, dar a conocer y preservar la flora autóctona argentina.

\section{AGRADECIMIENTOS}

Al personal de la Biblioteca de la Fundación Miguel Lillo por brindar el acceso a la información. A la curadora del Herbario Fanerogámico (LIL) por proporcionar el material que ilustra este artículo.

\section{BIBLIOGRAFÍA}

Castellanos, A. (1931). Lillo Miguel. Noticia necrológica. Physis 10: 427-431.

Dabbene R. y Lillo M. (1913). Description de deux nouvelles espèces d'oiseaux de la République Argentine. Anales del Museo Nacional de Historia Natural. Buenos Aires 24: 187-194.

Hauman, L. y Vanderveken G. (1917). Catalogue des Phanérogames de l'Argentine. I. Gymnospermes et Monocotylédones. Anales del Museo Nacional de Historia Natural. Buenos Aires 29: 1-351.

Hicken, C. (1922). Plantae Vattuonei. Darwiniana 1: 95-153.

Lillo, M. (1888). Flora de la provincia de Tucumán. Boletín de la Oficina Química Municipal de Tucumán I: 55-115.

Lillo, M. (1889). Flora de Tucumán. Herbier-Expos. Univers. Inter. De París: 341356.

Lillo, M. (1889). Apuntes sobre la fauna de la provincia de Tucumán. Enumeración y descripción de las especies animales indígenas con las costumbres y daños o beneficios que ocasionan las más características. Boletín de la Oficina Química Municipal de Tucumán II: 55-115. 
Lillo, M. (1902). Enumeración sistemática de las aves de la Provincia de Tucumán. Anales del Museo Nacional de Buenos Aires, VIII: 169-219.

Lillo, M. (1905). Fauna Tucumana. Aves. Rev. Letras y Cienc. Sociales, Tucumán.

Lillo, M. (1909). Notas Ornitológicas. Apuntes de Historia Natural: 21-26, 41-44.

Lillo, M. (1910). Contribución al conocimiento de los árboles de la Argentina, 1127.

Lillo, M. (1916). Flora de la Provincia de Tucumán. Gramíneas. Publicaciones hechas por el gobierno de Tucumán con motivo del Centenario de 1916: 1-63.

Lillo, M. (1917). Segunda contribución al conocimiento de los árboles de la Argentina. Universidad de Tucumán, Museo de Ciencias Naturales: 1-69. Ibid. (1924): $1-55$.

Lillo, M. (1919). Las Asclepiadáceas Argentinas. Physis IV: 410-437.

Lillo, M. (1917). Reseña fitogeográfica de la Provincia de Tucumán. Primera Reunión Nacional de la Sociedad Argentina de Ciencias Naturales: 210-232, incluye 1 mapa y 15 láminas.

Lillo, M. (1920). Las Asclepiadáceas de la República Argentina. Rev. Est. Univ. Tucumán: 113-118. Physis V (1921): 112-116.

Lillo, M. (1937). Catálogo de las Acantáceas argentinas. Lilloa 1: 21-66.

Molfino, J. F. (1937). Miguel Lillo (1862-1931). Lilloa 1: 7-19.

Peña de Bascary, S. (2014). Federico Schickendantz, Un científico investigador y vehemente. Revista de la Junta de Estudios Históricos de Tucumán, n ${ }^{\circ}$ 14. 124 pp.

Torres, A. (1958). Lillo, vida de un sabio. Publicación $\mathrm{N}^{\circ} 771$. Universidad Nacional de Tucumán. 335 pp.

Torres, A. (2006). Una historia de Lillo para jóvenes. Tucumán, Fundación Miguel Lillo. 84 pp. 